\title{
Convivencia sin violencia en una escuela pública del Perú
}

\section{Coexistence without violence in a public school in Peru}

DOI: $10.46932 / \mathrm{sfjdv2n2-165}$

Received in: March 1st, 2021

Accepted in: May 30th, 2021

\author{
Mg. Magaly Analí Galindo-Kimura \\ Filiación: Universidad César Vallejo \\ Correo: missmagaki@gmail.com \\ Dra. Isabel Menacho Vargas \\ Filiación: Universidad César Vallejo \\ Correo: isabelmenachov@gmail.com
}

Mg. Edith Mercedes Huertas Rueda

Filiación: Universidad Nacional José Faustino Sánchez Carrión

Correo: edithhuertas@gmail.com

Dr. Máximo Guillermo Valentín Montes

Filiación: Universidad Nacional Federico Villarreal

Correo: valedocente@gmail.com

\begin{abstract}
RESUMEN
La investigación fue realizada en torno a la convivencia escolar. Actualmente, los docentes interactúan con un gran porcentaje de estudiantes proceden de hogares carentes de valores donde existen agresiones de toda índole convirtiéndose en cotidiano, lo cual aflora en el aula. Los profesionales desconocen las leyes que protegen a los niños y adolescentes en el sector educación generando que los estudiantes se vean vulnerados. Por tal razón, se busca determinar que tanto mejora la convivencia escolar con una buena gestión desde la propia ley. El estudio se realiza desde un enfoque cuantitativo, tipo básico, diseño descriptivo, de corte transversal. Se buscó conocer los niveles de convivencia sin violencia en una institución escolar pública encontrando como resultado que el 48,3\% de docentes mencionan que existe una convivencia democrática, por otro lado, el $45 \%$ de docentes señalan que se encuentran en un nivel medio, mientras que el \% 6,7 en un nivel bajo.
\end{abstract}

Palabras clave: Política educativa, convivencia, violencia, democracia.

\begin{abstract}
The research was carried out around school coexistence. Currently, teachers interact with a large percentage of students come from homes devoid of values where there are attacks of all kinds, becoming everyday, which surfaces in the classroom. Professionals are unaware of the laws that protect children and adolescents in the education sector, causing students to be violated. For this reason, it seeks to determine how much school coexistence improves with good management from the law itself. The study is carried out from a quantitative approach, basic type, descriptive design, cross-sectional. It was sought to know the levels of coexistence without violence in a public-school institution, finding as a result that $48.3 \%$ of teachers mention that there is a democratic coexistence, on the other hand, $45 \%$ of teachers indicate that they are at an average level, while $6.7 \%$ at a low level.
\end{abstract}


Keywords: Educational policy, coexistence, violence, democracy.

\section{INTRODUCCIÓN}

Perú es un país con altos índices de violencia por las múltiples evidencias encontradas, Pedró (2020) describe situaciones negativas en cuanto a la convivencia familiar y social, más aún, aunado a una práctica educativa poco afectiva y empática que es preocupante en los últimos años. En ocasiones los acompañantes tutores no se han percatado del incremento de casos de agresiones en el hogar cometido por familiares. Asimismo, Cluver, Shenderovich, Meinck, Berezin, Doubt, Ward, Parra-Cardona, Lombard, Lachman, Wittesaele, Wessels, Gardner, \& Steinert (2020), refieren que las escuelas no están exentas de violencia entre pares, puesto que, en los últimos años, se ha incrementado las cifras según las estadísticas de la World Health Organization y Unicef que muestran la violencia constante entre compañeros de aula mediante las redes sociales, los cuales han incrementado los informes a nivel mundial sobre este tipo de casos.

Las investigaciones realizadas por Unesco manifiestan que existen conflictos recurrentes en las escuelas que están afectando directamente a los niños, niñas y jóvenes estudiantes y de paso al cuerpo docente. Esta problemática ya no solo incide en las aulas, sino que de una u otra forma transciende a la realidad social. Sin embargo, es erróneo pensar que una sociedad imita lo que pasa en las escuelas, por el contrario, son estas reproductoras de los comportamientos familiares y sociales tal como lo manifiestan Pacheco (2018); Bohórquez, Chaux, \& Serrano (2017); Chávez (2017); Trucco \& Inostroza (2017).

En consecuencia, si tenemos comunidades infectadas de violencia, entonces los niños, niñas y adolescentes tienen como primera opción, resolver las discrepancias de forma negativa recurriendo a la agresión verbal, exclusión, psicológica, física y hasta sexual. En su último informe, la Unesco (2020) afirma toda actitud de violencia afecta la salud mental de las personas y tiene como consecuencia el bajo rendimiento escolar, siendo más propensos a desertar de la educación formal. Heekes, Kruger, Lester, \& Ward (2020).

Grajales, Ceballos, Asprilla y Jaramillo (2019), encontraron que en las instituciones educativas donde los niños y jóvenes son víctimas de violencia intrafamiliar de parte de las figuras significativas manifiestan algunas conductas violentas en la escuela, teniendo como resultado problemas para interactuar entre estudiantes y con los docentes.

Según la Encuesta Nacional de Relaciones Sociales (2019), el 14.7\% fueron victimados por sus compañeros de clases, por otro lado, el 1,7\% de estudiantes informaron que habían sido víctimas de acoso escolar por parte de un docente y el 5,5\% de docentes informaron que un estudiante le había hecho bullying a otro estudiante. Otro resultado, emitido por el Ministerio de Educación del Perú (2019), señala 
que existe una mayor incidencia en situaciones de acoso en forma de burlas, apodos e insultos entre alumnos, en comparación de agresiones físicas directas, las cuales son de menor incidencia, los cuales fueron repostados en el portal especializado en casos de violencia escolar del SiseVe. También reportaron 36, 562 casos de violencia dentro de las escuelas y entre estudiantes entre los años 2013 al 2019. Asimismo, los casos de violencia cometidos de parte del personal educativo a estudiantes y viceversa fue dado en menor grado. Por tal razón, se centra una propuesta de trabajo del equipo responsable de la convivencia, del equipo directivo y del consejo educativo institucional de la convivencia democrática para afrontar situaciones de violencia existente. Carro-Olvera \& Lima-Gutiérrez (2020).

Desde años atrás, las medidas de previsión son complejas y enfocadas al sistema legal, pues estas según la comunidad educativa se perciben teóricamente, de otro modo, para que la Ley 29719 (2011), pueda desarrollarse efectivamente en un corto tiempo, se necesita interiorizar maximizando aspectos de intervención y prevención. Cabe resaltar que, las investigaciones mostraron que los resultados son mejores a largo plazo, ya que se consolidan los mecanismos prevención y de formación de conductas sociales. Las políticas de convivencia escolar han constatado ser un instrumento oportuno y eficaz como estrategia, por eso, se recomendó garantizar la aplicación efectiva de la ley, siguiendo el marco del cumplimiento del objetivo de desarrollo sostenible para acabar con toda forma de violencia que atente contra la niñez y adolescencia.

Hillis, Mercy, Amobi, \& Kress (2016) realizaron una investigación sobre la exposición de niños a la violencia a nivel regional y global donde se obtuvieron datos por grupo etario y país. Los resultados revelaron muchos hallazgos rotundos sobre la prevalencia de la violencia contra niños en los últimos años. Las estimaciones realizadas en 96 países que un mínimo de 50\% de niños entre 2 y 17 años experimentaron algún tipo de violencia. Según la estadística realizada, se sintetizó los datos utilizando un método de triangulación para la generación de resultados derivadas de promedios ponderados por grupos específicos. También la implementación de los objetivos del programa, incluyendo el desarrollo de procedimientos para una vigilancia poblacional ampliada contra la violencia en niños que mejoró la comunicación y la coordinación de nuevas asociaciones entre los agentes y toda la comunidad acortando las brechas existentes.

En las investigaciones tomadas de las escuelas de Asia Oriental se visualizó un ambiente diferente que está fuertemente marcada por un lado oscuro como es la intimidación, que ha conllevado a altas tasas de suicidio juvenil. Los sociólogos de la juventud japonesa definen estos episodios como "pánicos morales". A su vez es recurrente el acoso escolar que están determinados, al menos en parte, por diferencias profundas sociales y la autoestima. Para Rappleye y Komatsu, (2020), este análisis contribuye 
al trabajo reciente que ha desafiado los estereotipos persistentes de la educación, donde los jóvenes al sentirse impotentes frente al bullying optan por tomar decisiones fatales.

Treviño y González (2020), en su modelo teórico determinaron una relación entre los indicadores de participación, atención, sanciones y condiciones para una buena convivencia escolar donde los maestros promueven que se apliquen y cumplan estas condiciones en el aula de clases, siendo cruciales para la mejora de la convivencia. Los resultados hallados demostraron que al involucrarse los docentes y las autoridades educativas fue un hecho trascendental en la investigación.

La Universidad de Cantabria afirma que las políticas y prácticas educativas son primordiales para una convivencia escolar. Los resultados obtenidos demostraron que una buena convivencia facilita la formación académica de los educandos, pues favorece la cimentación de una ciudadanía participativa que facilita la convivencia democrática. Education Letter (2020).

Edurne, Martínez, Fonseca, Tamayo y Acosta (2019) realizaron una investigación denominada acoso escolar con el fin de evaluar métodos y técnicas de intervención en un grupo de estudiantes. Se realizó utilizando herramientas que midan la capacidad y habilidades frente al acoso escolar. Finalmente se demostró que existe una necesidad de mejorar y extender las intervenciones en función de cada grupo formativo teniendo en cuenta su realidad situacional.

En otra investigación se menciona que las variables sobre exclusión, problemas de aprendizaje, conflictos interpersonales, malas prácticas docentes y violencia entre estudiantes son asociadas a la convivencia escolar, considerando que los resultados demuestran una alta influencia, como característica más destacada entre convivencia y violencia escolar presente en muchas escuelas de Latinoamérica. Andrades-Moya, Castrillón-Correa \& Pérez-Álvarez (2020).

Carbajal (2018) y De Vasconcelos (2017), refieren en su investigación que, para superar la violencia escolar, los castigos no funcionan; en cambio, al escuchar a las víctimas se construyen relaciones más sólidas entre los protagonistas y la escuela permitiendo experimentar nuevos horizontes de relaciones humanas en los contextos marcados por la pobreza y la violencia.

Bonilla; Rosario; Rivera; Muñoz; y Rivera. (2020) realizaron una investigación donde se construyó una escala que mide las actitudes violentas en estudiantes. La meta de esta investigación fue construir una tabla de indicadores que midan la intimidación y el maltrato verbal, el sometimiento y la humillación causada por escolares, buscando comprender las actitudes de los agresores involucrados en esta problemática. Los resultados mostraron la validez y confiabilidad obtenidos en los datos mediante pruebas psicométricas (AVE-E) que miden actitudes de violencia escolar en adolescentes de aproximadamente 15 años de edad, en donde se planteó programas para controlar la violencia dentro de una institución educativa mediante promoción, prevención e intervención oportuna. 
Para los investigadores Freire y Miranda, (2018), la violencia escolar es un factor que influye en la convivencia escolar. Por otro lado, mencionan que contar con un ambiente adecuado propicia el desarrollo de los estudiantes y garantiza una educación de calidad en la zona altoandina, que es una región con altos índices de pobreza, analfabetismo y desnutrición infantil. Los resultados mostraron que los niños varones entre 8 a 12 años son más propensos a ser víctimas de castigos corporales por su bajo rendimiento en algunas materias, existiendo un rendimiento diferenciado entre niños y niñas a causa de la violencia.

Para los investigadores Sampén, Aguilar y Tójar (2017), al aplicar un programa de mejora de actitudes de convivencia escolar basado en la competencia social e interpersonal, los investigados mejoraron sus actitudes entre pares mediante el uso del diálogo, comprensión, empatía y expresión de sentimientos afianzando los lazos de amistad y solidaridad durante situaciones conflictivas. Se utilizó un enfoque de investigación mixto y el objetivo fue prevenir el maltrato escolar. Los resultados del programa contribuyeron a potenciar los aprendizajes y la competencia social.

Sharma, Nam, Kim \& Kim. (2016) resaltaron que al presenciar la violencia entre padres en casa y el ser víctimas de intimidación es común para muchos niños y adolescentes en muchos lugares. Este estudio examinó el papel de presenciar la violencia familiar y la participación de adolescentes en peleas físicas, estas últimas se asociaron con mayores probabilidades. Crear un entorno no violento tanto en el hogar como en la escuela sería una estrategia eficaz para reducir las peleas entre la población de jóvenes adolescentes.

El presente trabajo de investigación buscó desarrollar y mejorar la convivencia para estudiantes, maestros y directivos teniendo como fuente de justificación la aplicación de la ley 29719 en una institución escolar y la convivencia democrática. Dentro de lo cual, la revisión del material teórico nos permitió mediante un acto reflexivo, postular estrategias de como promover mejoras de las relaciones interpersonales entre los mismos estudiantes, la plana docente y personal administrativo en una institución escolar. El objetivo de la presente investigación permitió determinar el nivel de gestión de la convivencia escolar, utilizando las normas que promueven la convivencia sin violencia en una escuela pública.

\section{MARCO TEÓRICO}

Para abordar la problemática que enfrentan en este momento las escuelas, en cuanto a la convivencia sin violencia, han surgido diversas concepciones desde los años noventa en adelante. Los reportes de las investigaciones muestran que al tener una diversidad de caracteres individuales, aunado a las condiciones de madurez biopsicosocial, se afrontan grandes desafíos debido a la complejidad de interacciones sociales, como lo definen Velázquez-Guzmán \& Lara-Rosano (2017). En estudios comparados, los factores externos impactan en la dinámica escolar, como son la exclusión y el rezago 
escolar que han influido en la interacción interpersonal y académica, resultando que el tema de convivencia sin violencia es un asunto central e importante del proceso educativo.

Por lo tanto, es necesario una cultura armónica enfatizado por Galtung. (1996) quien, en sus reflexiones sobre dimensiones éticas, socioculturales y legales de una cultura de paz, subraya la necesidad de potenciar estrategias para controlar el comportamiento de estudiantes, enfatizando el orden y respeto para el desarrollo de las sociedades.

En los informes de Unesco se pretende que las sociedades conozcan y practiquen los pilares de aprendizaje propuestos por las comisiones de educación como es el aprender a convivir juntos. Es un tema preocupante en la actualidad. Asimismo, se indicó que es necesario la construcción y fortalecimiento de la convivencia democrática para desarrollar valores como el respeto hacia los demás, la valoración de la cultura propia. Reforzar valores espirituales y morales implementando proyectos educativos en unión de los agentes sociales que promuevan la resolución de conflictos pacíficamente. Fierro \& Carbajal (2019). A pesar que, los problemas de convivencia son frecuentes en las escuelas, no se puede tener una visión negativa generalizada de la convivencia escolar.

Es importante dentro del trabajo docente tener en cuenta las normas y disposiciones emanadas por el ministerio de educación, en tanto provee un camino de acciones necesarias, como es el caso de la ley 29719 que promueve la convivencia democrática en las escuelas. El objetivo que persigue es establecer los mecanismos para evitar el acoso, la atemorización y cualquier acto considerado como hostigamiento para mellar los problemas suscitados, tal como se manifiesta en el artículo 1 y 2 ley que regula el acoso escolar en cualquiera de sus modalidades antes descritas.

Una fuente fundamental es el proyecto Educativo Nacional al 2036 que se relaciona con la promoción de la convivencia escolar en las escuelas y más aún, siendo coherentes con las políticas que manifiestan el interés de convertir las instituciones educativas en espacios organizados y acogedores que propicien la cohesión de la comunidad educativa teniendo como fin una convivencia armónica, grata y productiva de cara al fututo. Tafur \& Suaréz (2014).

En los diferentes contextos socio ambientales en general, los estudiantes asumen sus roles como ciudadanos a través de las diferentes experiencias y actividades escolares que participan. Según Losito; Damiani; Agrusti \& Schulz. (2018), las principales experiencias que tienen los estudiantes en la escuela permiten desarrollar actitudes y valores, puestos que, los resultados de aprendizaje no dependen de procesos de enseñanza solamente, sino de experiencias en general que ocurren a través de la participación e involucramiento o interacción social. Cabe resaltar que, los procesos participativos en la escuela desarrollan la competencia ciudadanía y familiar de acuerdo con el marco de evaluación del ICCS, quienes fomentan la educación cívica y ciudadana. Series (2020). 
Una situación en común sostiene Eckenrode, Laird \& Doris (1993), que los niños maltratados se desempeñaron significativamente por debajo de sus compañeros no maltratados en las pruebas estandarizadas obteniendo más probabilidades de repetir un grado. Aquellos niños violentados también tuvieron más trastornos cognitivos y suspensiones disciplinarias. Por otra parte, los niños abandonados mostraron los peores resultados en el rendimiento académico, en cambio, los maltratados físicamente mostraron mayores problemas de disciplina.

\subsection{APLICACIÓN DE ESTRATEGIAS QUE PROMUEVEN LA CONVIVENCIA SIN VIOLENCIA}

Una de las actividades para la solución de conflictos es la estrategia cooperativa, para Zamora (2020) permite adquirir competencias como son la escucha asertiva, respeto a las opiniones de los demás, la solución de conflictos mediante la expresión cortés de diversas opiniones. En este sentido, los estudiantes buscaran potenciar sus habilidades socio emocionales para la resolución de conflictos mediante un trabajo personal y en conjunto para la solución de conflictos existentes. Es necesario reforzar la capacidad de auto regulación emocional como forma de resolver los conflictos como lo sostiene Galvis (2014). Por otro lado, el uso de la técnica del debate como socialización contribuyó en muchos casos a la mejora de ambientes escolares sin violencia recomendado por Álvarez-Cañizo, Cueva, Cuetos \& SuárezCoalla (2020).

\subsection{GESTIÓN DE LAS FUNCIONES DE LA DIRECTIVA INSTITUCIONAL}

Según Andrades, Castrillón, y Pérez (2020), para fortalecer la gestión participativa del equipo directivo en convivencia escolar se debe tener en cuenta la importancia del trabajo cooperativo siendo de vital importancia la competencia del liderazgo según Minedu (2018), así como, la aceptación mutual, la cooperación y el respeto a las diferencias; también la valoración de la identidad cultural y los derechos personales se basan en las relaciones humanas. Por tal razón, es necesario el desarrollo de un liderazgo dentro de la formación de los directivos, siendo evidente el reconocimiento de prácticas exitosas en las escuelas que trabajan desde una visión distribuida cooperativamente entre los docentes y el equipo directivo institucional. Sandoval, Pineda, Bernal, y Quiroga, (2020).

Analizando el reglamento de la ley 29719 en el artículo 11, define que las funciones del director son garantizar la elaboración de un plan de convivencia escolar. Además, debe supervisar los procedimientos y medidas correctivas que promuevan la convivencia sin violencia en las escuelas. Finalmente, debe apoyar las acciones que realiza el equipo responsable de convivencia democrática, comunicando y rindiendo cuentas de los logros obtenidos a la comunidad educativa. (Cea, García, Nail Kröyer y Paredes (2018). 


\subsection{GESTIÓN DE LAS FUNCIONES DEL CONCEJO EDUCATIVO INSTITUCIONAL}

Para Andino (2018), La capacitación constante de los docentes del concejo educativo institucional es imprescindible desde cualquier punto de vista. El asumir estas funciones permitirá enfrentar situaciones conflictivas en la escuela. Por tal motivo, es sumamente necesaria dado el creciente incremento de casos, pues permite identificar y gestionar la violencia estudiantil. Tafur y Suarez (2014), afirma que favorece la construcción y fortalecimiento de una convivencia escolar armónica cuando cada miembro asume los roles de forma consciente y responsable.

Dentro del análisis del reglamento de la ley 29719 en el artículo $\mathrm{N}^{\circ} 4$, estipula que el concejo educativo institucional realizará las funciones de control y ejecución del proyecto de convivencia democrática. Durante el desarrollo se promoverá la participación de aliados estratégicos y/o profesionales que apoyen acciones planificadas para resolver las controversias y conflictos que surjan de manera participativa y funcional.

\subsection{APLICACIÓN DE LA LEY 29719 QUE PROMUEVE LA CONVIVENCIA SIN VIOLENCIA}

Según lo señalan Chaparro et al., (2015), la aplicación de normas o leyes no ha sido pertinente, pues, algunos testigos o víctimas no reportan los hechos de violencia por temor o indiferencia; teniendo como reto lograr prácticas inclusivas y equitativas para formar una sociedad más justa a priori. Existe un desafío teórico y metodológico dentro del contexto escolar puesto que se requiere evaluar los planes de convivencia dentro de la escuela. En muchos casos no se realiza un consolidado al fin de año que permita conocer si realmente la aplicación del plan de convivencia escolar tuvo el éxito deseado.

\section{METODOLOGÍA}

La investigación fue de tipo básica de nivel descriptivo, tal como lo manifestaron Hernández y Mendoza. (2018) al indicar que la investigación descriptiva especifica las propiedades, las características y los perfiles de personas, comunidades, procesos, objetos o cualquier otro fenómeno de un grupo o población en un análisis. Por el diseño, la investigación fue no experimental, pues se fundó en las observaciones de los hechos en estado natural, sin la intervención o manipulación del investigador. Asimismo, el diseño fue descriptivo simple, además transversal, porque se aplicó el instrumento en una sola oportunidad.

La población estuvo formada por 60 docentes entre nombrados y contratados, se utilizó el censo. Para recopilar los datos se empleó la encuesta y como instrumento el cuestionario que constó de 40 ítems. Se trabajó con la escala Likert, la cual permitió conocer la percepción de los docentes. El análisis de resultados se realizó a través de la estadística descriptiva y SPSS versión 25. 


\section{RESULTADOS Y DISCUSIÓN}

En una muestra de 60 docentes se aplicó los instrumentos para conocer los niveles de la convivencia sin violencia encontrándose

Tabla 1. Aplicación de estrategias que promueven la convivencia sin violencia.

\begin{tabular}{lcc}
\hline Niveles & Frecuencia & Porcentaje \\
\hline Bajo & 3 & 5,0 \\
Medio & 29 & 48,3 \\
Alto & 28 & 46,7 \\
\hline Total & 60 & 100,0 \\
\hline
\end{tabular}

En la tabla 1, los resultados de la investigación revelan que la aplicación de estrategias propuestas de la ley 29719 en un nivel medio alto, precisando que existe un trabajo en conjunto de los actores educativos en dicho centro, tal como lo mencionó Jaycox et al. (2020). Una estrategia es construir relaciones más sólidas entre las familias fortalece las relaciones humanas permiten experimentar nuevos horizontes en contextos marcados por la pobreza y la violencia. Carbajal, (2018) y de Vasconcelos, (2017). Desarrollar la competencia ciudadanía en las familias y la comunidad, a través de una estrategia participativa como mencionó Schulz et al., (2016)

Tabla 2. Gestión de las funciones de la directiva institucional

\begin{tabular}{lcc} 
Niveles & Frecuencia & Porcentaje \\
\hline Bajo & 13 & 21,7 \\
Medio & 22 & 36,7 \\
Alto & 25 & 41,7 \\
\hline Total & 60 & 100,0 \\
\hline
\end{tabular}

En la tabla 2, los resultados muestran que los encargados de la dirección aplican la ley 29719 que promueve la convivencia sin violencia entre toda la comunidad educativa teniendo un alta de gestión de la norma, favoreciendo la convivencia escolar en sus distintos contextos.

En suma, la gestión participativa ameritó una promoción de una cultura de trabajo cooperativo, generación de oportunidades y de participación entre sus distintos actores dentro de un clima de confianza. Edurne, Martínez, Fonseca, Tamayo y Acosta (2019) propusieron que cada grupo formativo y directivo mejorará la intervención de acuerdo a su función asumida. También, según Andrades, Castrillón y Pérez (2020) la perfeccionamiento de las aptitudes positivas como el liderazgo y el trabajo cooperativo fortalecen la gestión participativa de los directivos.

Tabla 3. Gestión de las funciones del concejo educativo institucional

\begin{tabular}{lcc} 
Niveles & Frecuencia & Porcentaje \\
\hline Bajo & 12 & 20,0 \\
Medio & 26 & 43,3 \\
Alto & 22 & 36,7 \\
\hline Total & 60 & 100,0 \\
\hline
\end{tabular}


En la tabla 3, los resultados de la investigación muestran que las funciones del concejo institucional se ubican en el nivel medio con un 43,3\% de aceptación por parte de los docentes encuestados, sobre la aplicación de ley que promueve la convivencia sin violencia. Para los autores es necesario que los profesionales educativos realicen un seguimiento frente al acoso.

Andino (2018) la capacitación del concejo educativo institucional busca enfrentar situaciones conflictivas en la escuela identificando los casos de violencia que surjan en la institución, ya que los jóvenes al sentirse impotentes frente al bullying optan por tomar decisiones fatales como mencionan Rappleye y Komatsu, (2020) así como lo sustentan también Treviño y González, (2020) que los docentes y las autoridades educativas son piezas transcendentales en este tema.

Tabla 4. Aplicación de la Ley 29719 que promueve la convivencia sin violencia

\begin{tabular}{lcc} 
Niveles & Frecuencia & Porcentaje \\
\hline Bajo & 4 & 6,7 \\
Medio & 27 & 45,0 \\
Alto & 29 & 48,3 \\
\hline Total & 60 & 100,0 \\
\hline
\end{tabular}

Los resultados de la investigación señalan que la aplicación de la ley 29719 que promueve la convivencia sin violencia fue conocida y practicada en la institución educativa, tanto por directivos, consejo educativo, los docentes y estudiantes, haciendo cumplir los valores institucionales y las normas de convivencia escolar. Sin embargo, en otros contextos Andrades J, (2020) las malas prácticas docentes, exclusión y violencia escolar han ifluido en la convivencia escolar siendo un desafío para la comunidad educativa según lo sostienen Chaparro et al., (2015).

\section{CONCLUSIONES}

La investigación se realizó mediante el análisis de la política educativa sobre convivencia escolar en una escuela del Perú. En primer lugar, se analizó las características de los instrumentos de política educativa y, en segundo lugar, desarrollar la puesta en práctica de normas que promueve la convivencia sin violencia, la cual, constituye un componente central según lo diseñado e implementado en relación a la convivencia escolar.

Las políticas educativas deben ser evaluadas en la misma escuela por parte de organismos supervisores, que mediante propuestas de acciones pedagógicas se construyan en conjunto recursos de solución desde la realidad problemática, de cara a permitir consolidar una identidad al interior de la escuela que impliquen los conocimientos de sí mismos, los sentimientos, las actitudes y los valores Serey \& Zuñiga (2021). Como reto futuro, los equipos directivos, docentes y administrativos necesitan fortalecer su formación, analizando y reflexionado las propuestas educativas actuales y su implicancia en la escuela.

Mejorar la convivencia escolar requiere de nuevas propuestas didácticas, donde se comprometan todos los integrantes de la escuela a formar parte de un trabajo cooperativo realizando nuevas propuestas 
que fomenten la convivencia escolar que a un mediano plazo pueda impactar positivamente en las actitudes y los valores mediante una política de paz y derechos humanos. Álvarez y Pérez (2019) impulsar estrategias de coaching educativo que promueva el respeto mutuo, la tolerancia, la empatía y la inclusión, las cuales constituyen el marco normativo para una buena convivencia escolar. 


\section{REFERENCIAS}

Álvarez-Cañizo, M., Cueva, E., Cuetos, F., \& Suárez-Coalla, P. (2020). Reading fluency and reading comprehension in spanish secondary students. Psicothema, 32(1), 75-83. https://doi.org/10.7334/psicothema2019.196

Álvarez-Maestre, A. J., \& Pérez-Fuentes, C. A. (2019). Educación para la paz: Aproximación teórica desde los imaginarios de paz. Educación y Educadores, 22(2), 277-296. doi:10.5294/edu.2019.22.2.6

Andino, R. A. (2018). Capacitación docente: Pilar para la identificación y gestión de la violencia escolar. Alteridad, 13(1), 108-119.

Andrades-Moya, J., Castrillón-Correa, E. M., \& Pérez-Álvarez, E. O. (2020). Family ( ies ) in studies about school coexistence in Chile: a systematic review. 14(3), 424-433. https://doi.org/10.11591/edulearn.v14i3.16582

Andrades Moya, J. (2020). Convivencia escolar en Latinoamérica: Una revisión bibliográfica. Revista Electrónica Educare, 24(2), 1-23. https://doi.org/10.15359/ree.24-2.17

Bohórquez Correa, R. I., Chaux Real, Y. N., \& Serrano Figueroa, M. P. (2017). El conflicto en la convivencia escolar: Creencias y prácticas de los estudiantes, padres de familia y docentes de una Institución Educativa Distrital. Actualidades Pedagógicas, 1(70), 29-49. https://doi.org/10.19052/ap.4087

Bonilla Asalde, C., Rosario Quiroz, F. J., Rivera, O., Muñoz, H., \& Rivera, I. C. (2020). Construcción de una escala de actitud hacia la violencia escolar en adolescentes del Callao, Perú. Fides Et Ratio, 19, 4976.

Carbajal, M. P. (2018). Building democratic convivencia (peaceful coexistence) in classrooms: case studies of teaching in mexican public schools surrounded by violence. 356. http://hdl.handle.net/1807/89835

Carro-Olvera, A., \& Lima-Gutiérrez, J. A. (2020). Política educativa, violencia y convivencia escolar. La experiencia en dos escuelas. Ensaio: Avaliação e Políticas Públicas Em Educação, 28(107), 314-334. https://doi.org/10.1590/s0104-40362019002701955

Cea Anfossi, P., García Ramírez, Lady, Nail Kröyer, O., \& Paredes Ulloa, D. (2018). Gestión participativa en directivos para el abordaje de la convivencia escolar en instituciones educativas municipalizadas. Revista Iberoamericana de Educación, 76(2), 153-184. https://doi.org/10.35362/rie7623039

Chaparro Caso-López, A. A., Caso Niebla, J., Fierro Evans, M. C., \& Díaz López, C. (2015). Desarrollo de un instrumento de evaluación basado en indicadores de convivencia escolar democrática, inclusiva y pacífica. XXXVII, 20-41.

Chávez González, M. L. (2017). La violencia escolar desde La perspectiva infantil en El altiplano Mexicano. Revista Mexicana de Investigacion Educativa, 22(74), 813-835.

Congreso de la República del Perú. (2011). Ley $N^{\circ} 29719$, Ley que promueve la Convivencia Escolar en las Instituciones Educativas. In El Peruano (pp. 445297-445298). https://leyes.congreso.gob.pe/Documentos/Leyes/29719.pdf 
Cluver, L., Shenderovich, Y., Meinck, F., Berezin, M. N., Doubt, J., Ward, C. L., Parra-Cardona, J., Lombard, C., Lachman, J. M., Wittesaele, C., Wessels, I., Gardner, F., \& Steinert, J. I. (2020). Parenting, mental health and economic pathways to prevention of violence against children in South Africa. Social Science and Medicine, 262(July), 113194. https://doi.org/10.1016/j.socscimed.2020.113194

De Vasconcelos, I. C. O. (2017). Aprender a conviver, sem violência: O que dá e não dá certo? Ensaio, 25(97), 897-917. https://doi.org/10.1590/S0104-40362017002501180

Eckenrode, J; Laird, M \& Doris, J. (1993). School performance and disciplinary problems among abused and neglected children. Developmental Psychology, 29(1), 53-62.

Education Letter; Atlanta. (2020). Education; New Findings from University of Cantabria in the Area of Education Described ( Strengths, weaknesses and challenges in the management of school coexistence : significance of the management teams of primary and secondary education schools ). 1-4.

Edurne, A., Martinez, F., Fonseca, R., Tamayo, Y., \& Acosta, M. (2019). Bullying o Acoso escolar . Creatividad frente al rol de adolescentes Bullying or bullying . Creativity versus the role of teenagers Bullying ou bullying . Criatividade versus papel dos adolescentes. Multimed. Revista Médica. Granma Version, 23(6), 1202-1215. http://scielo.sld.cu/pdf/mmed/v23n6/1028-4818-mmed-23-06-1202.pdf

Enares. (2019). Encuesta Nacional Sobre Relaciones Sociales. In Instituto Nacional de Estadística e Informática.http://www.ncbi.nlm.nih.gov/pubmed/15003161\%5Cnhttp://cid.oxfordjournals.org/lookup/ doi/10.1093/cid/cir991\%5Cnhttp://www.scielo.cl/pdf/udecada/v15n26/art06.pdf\%5Cnhttp://www.scopu s.com/inward/record.url?eid=2-s2.0-84861150233\&partnerID=tZOtx3y1

Fierro, C., \& Carbajal, P. (2019). Convivencia Escolar: Una revisión del concepto. Psicoperspectivas. Individuo $\quad \mathrm{y} \quad$ Sociedad, $18(1)$, 9-27. http://www.psicoperspectivas.cl/index.php/psicoperspectivas/article/view/1486

Freire, S., \& Miranda, A. (2018). Avances de Investigación educación y aprendizajes. Grade, 1(1), 1-230. Galtung, J. (1996). Peace and conflict research in the age of the cholera: ten pointers to the future of peace studies. International Journal of Peace Studies, 1(1), 25-36. Retrieved May 14, 2021, from http://www.jstor.org/stable/45038019

Galvis, R. I. (2014). Las neuronas espejo y el desarrollo de la empatía frente a la agresión y el conflicto en la escuela. Praxis pedagógica, 15,43-53.

Grajales Guzmán, C., Ceballos Ortiz, D., Asprilla Atencia, E., \& Jaramillo Valencia, B. (2019). Violencia intrafamiliar y pensamiento creativo: factores que inciden en la resolución de conflictos en la infancia. Actualidades Pedagógicas, 1(74), 127-139. https://doi.org/10.19052/ap.vol1.iss74.6

Hernández Sampieri, R., \& Mendoza Torres, C. P. (2018). Metodología de la investigación: las tres rutas cuantitativa, cualitativa y mixta . In Mc Graw Hill (Vol. 1, Issue Mexico). http://www.mhhe.com/latam/sampieri_mile

Hillis, S., Mercy, J., Amobi, A., \& Kress, H. (2016). Global prevalence of past-year violence against children: A systematic review and minimum estimates. In Pediatrics (Vol. 137, Issue 3). American Academy of Pediatrics. https://doi.org/10.1542/peds.2015-4079

Heekes, S. L., Kruger, C. B., Lester, S. N., \& Ward, C. L. (2020). A Systematic Review of Corporal 
Punishment in Schools: Global Prevalence and Correlates. Trauma, violence \& abuse, 1524838020925787. Advance online publication. https://doi.org/10.1177/1524838020925787

Jaycox, L., Hickman, L., Schultz, D., Barnes-Proby, D., Setodji, C., Kofner, A., Harris, R., Acosta, J., \& Francois, T. (2020). Reducing the Impact of Children's Exposure to Violence: Results of the National Evaluation of Safe Start Promising Approaches. Reducing the Impact of Children's Exposure to Violence: Results of the National Evaluation of Safe Start Promising Approaches. https://doi.org/10.7249/rb9575

Minedu, M. de E. del P. (2018). Escolar, La Prevención Y La Atención De La. 56. http://repositorio.minedu.gob.pe/bitstream/handle/MINEDU/6088/Lineamientos para la gestión de la convivencia escolar\%2C la prevención y la atención de la violencia contra niñas\%2C niños y adolescentes.pdf? sequence $=1 \&$ isAllowed $=\mathrm{y}$

Losito, B., Damiani, V., Agrusti, G., \& Schulz, W. (2018). Young People's Perceptions of Europe in a Time of Change: IEA International Civic and Citizenship Education Study 2016 European Report. Young People's Perceptions of Europe in a Time of Change: IEA International Civic and Citizenship Education Study 2016 European Report, 1-72. https://doi.org/10.1007/978-3-319-73960-1

Pacheco Salazar, B. (2018). Violencia escolar: la perspectiva de estudiantes y docentes. Revista $\begin{array}{lllll}\text { Electrónica de Investigación } & \text { Educativa, } & \text { 20(1), } & 112-127 .\end{array}$ http://www.scielo.org.mx/scielo.php?pid=S1607-40412016000100008\&script=sci_arttext

Rappleye, J., \& Komatsu, H. (2020). Is bullying and suicide a problem for East Asia's schools? Evidence from TIMSS and PISA. Discourse, 41(2), 310-331. https://doi.org/10.1080/01596306.2020.1711515

Sampén-Díaz, M. N., Aguilar-Ramos, M. C., \& Tójar-Hurtado, J. C. (2017). Educando la competencia social en Perú. Programa de prevención del maltrato escolar. Revista Electrónica de Investigación Educativa, 19(1), 46. https://doi.org/10.24320/redie.2017.19.1.1013

Sandoval, L.Y., Pineda, C., Bernal, R. y Quiroga, C. (2020). Los retos del director novel: formación inicial y liderazgo. Revista Complutense de Educación, 31(1), 117-126. https://doi.org/10.5209/rced.61919

Sharma, B., Nam, E. W., Kim, H. Y., \& Kim, J. K. (2016). The Influence of Witnessing Inter-parental Violence and Bullying Victimization in Involvement in Fighting among Adolescents: Evidence from a School-based Cross-sectional Survey in Peru. Journal of Lifestyle Medicine, 6(1), 27-35. https://doi.org/10.15280/jlm.2016.6.1.27

Serey, D., \& Zuñiga, P. (2021). Página 144. La Convivencia Escolar Post COVID 19: Una Propuesta Didáctica Desde El Coaching Educativo, 143-161.

Series G. (2020). International Civic and Citizenship Education Study. Gem, 17, 109-120.

Tafur, R., \& Saréz, G. (2014). La Convivencia en el Centro Educativo. Reflexiones desde Perú. La Convivencia En Los Centros Educativos de Educación Básica En Iberoamérica, 118-134. https://ddd.uab.cat/pub/llibres/2014/131430/Red_AGE_2014_web.pdf

Treviño Villarreal, D. C., \& González Medina, M. A. (2020). Involucramiento docente y condiciones del aula : una diada para mejorar la convivencia escolar en bachillerato Teacher involvement and classroom conditions : a dyad to improve school coexistence in baccalaureate. 38, 397-414. 
Trucco, D., \& Inostroza, P. (2017). Las violencias en el espacio escolar. 117. www.cepal.org/es/suscripciones

Unesco. (2020). Nuevo Día Internacional contra la Violencia y el Acoso en la Escuela, incluido el Ciberacoso. Autor. https://es.unesco.org/commemorations/dayagainstschoolviolenceandbullying

Velázquez-Guzmán, M. G., \& Lara-Rosano, F. (2017). Estrategias educativas para la convivencia escolar de los adolescentes. Editorial Académica Española, December. https://www.researchgate.net/publication/322136810_libro_estrategias_educativas_para_la_convivencia _escolar_de_los_adolescentes

Zamora, N. A. (2020). Influencia de estrategias cooperativas en la solución de conflictos escolares. Polo Del Conocimiento, 5(9), 148-161. https://doi.org/10.23857/pc.v5i8.1670 